\title{
Study of the RPA Pair-Correlation Function in GaAs-AlGaAs Parabolic Quantum Well Wires
}

\author{
J. B. B. da Cunha*, J. F. R. da Cunha ${ }^{\dagger}$, P. C. M. Machado*, F. A. P. Osório*†, and A. N. Borges*† \\ * Instituto de Física, Universidade Federal de Goiás, CP 131, 74001-970, Goiânia, GO, Brazil \\ ** Escola de Engenharia Elétrica, Universidade Federal de Goiás, CP 131, 74001-970, Goiânia, GO, Brazil \\ $\dagger$ Núcleo de Pesquisa em Física, Universidade Católica de Goiás, 74605-220, Goiânia, GO, Brazil
}

Received on 12 March, 2003

\begin{abstract}
The ground state intrasubband pair-correlation function for a quasi-one-dimensional electron gas confined in a GaAs- $\mathrm{Al}_{x} \mathrm{Ga}_{1-x}$ As parabolic quantum well wire within the Random-Phase Approximation (RPA) is calculated. We have considered two wires with subband energies separation $\hbar \Omega=2.0 \mathrm{meV}$ and $\hbar \Omega=2.5 \mathrm{meV}$. The dependence of the pair-correlation function on the electronic density was studied and the regions where the RPA approach cannot be used were precisely determined.
\end{abstract}

Recently much theoretical work has been done to the understanding of the properties of the plasmon collective excitation in quasi-one-dimensional (Q1D) semiconductor quantum well wires (QWW). In such systems, the electronic motion in the plane perpendicular to the wire extension is quantized in subbands. Experiments performed by Demmel et al. [1] have shown that the subband quantization effects are important to the understanding of the collective motion in quantum wires. Various theoretical methods have been utilized to calculate the collective excitations of electron gas in QWW and among them, the most common is the Random Phase Approximation (RPA). This approach, as is well known, does not consider the local field correction (LFC) and such simplification of the calculation can lead to negative values for the pair correlation function. It is a nonphysical result, since the pair correlation function, $g(x)$, give us the probability of a particle can be observed in a position $\mathrm{x}$ if other particle is in the origin of the coordinates, thus the acceptable values for this function must be positive.

The choice of the better approach to use for the calculation of the collective excitations is strongly dictated by the electronic density of the system. When the electronic density is sufficiently large the RPA approach describes the system properties satisfactorily. The electronic density from which the RPA gives non-negative values for the pair correlation function for small interparticle separation is defined as the critical density. For density values lower than the critical density the LFC must always be used [2-5].

In the present work we calculate the ground state intrasubband pair-correlation function for a Q1D electron gas present in a GaAs-AlGaAs parabolic quantum well wire. The Q1D electron gas in the parabolic QWW has a quasifree motion in the $x$-direction and a restrict motion in the plane $y-z$. Here we assumed that the confinement potential $V(y, z)$ can be written as a sum of the one-dimensional potentials $V(y)$ and $V(z)$. We employed a two-subband model at zero temperature. The confinement potential in the $z$-direction, $V(z)$, was considered much larger than the parabolic confinement potential $V(y)=\frac{1}{2} m \Omega y^{2}$, where $m$ is the electron effective mass of the semiconductor and $\hbar \Omega$ is the subband energy separation. Since the confinement in the $z$-direction is higher than the parabolic confinement, the energy separation between the subbands can be neglected and only the lowest subband in the $z$-direction is considered be occupied by electrons.

The pair-correlation function for the subband ijlm is given by the relation [6]

$$
g_{i j l m}(x)=1+\frac{1}{\pi \rho} \int_{0}^{\infty} d q_{x}\left[-\frac{\hbar}{\pi \rho} \int_{0}^{\infty} d \omega \operatorname{Im} \chi_{i j l m}\left(q_{x}, \omega\right)-1\right] \cos \left(q_{x} x\right)
$$

where $q_{x}$ and $\omega$ are the wave vector in the direction parallel to the wire and the frequency of the electron gas, respectively. The electron density is denoted by $\rho$, and $\operatorname{Im} \chi_{i j l m}\left(q_{x}, \omega\right)$ denotes the imaginary part of the RPA gen- eralized response function of the system, which can be written as, 


$$
\chi_{i j l m}\left(q_{x}, \omega\right)=\frac{\chi_{l m}\left(q_{x}, \omega\right)}{\delta_{i l} \delta_{j m}-V_{i j l m}^{C}\left(q_{x}\right) \chi_{l m}\left(q_{x}, \omega\right)}
$$

where the symbol $\delta i j$ is the Kronecker delta, $\chi_{l m}=P_{l m}$ if $l=m$ and $\chi_{l m}=P_{l m}+P_{m l}$ when $l \neq m . P_{l m}\left(q_{x}, \omega\right)$ is the RPA polarization function of the Q1D electron gas [6]. Finally, $V_{i j l m}^{C}\left(q_{x}\right)$ is the subband matrix element of the
Coulomb interaction that has the following expression,

$$
V_{i j l m}^{C}\left(q_{x}\right)=\frac{2 e^{2}}{\varepsilon} \int_{0}^{\infty} d k \frac{F_{i j l m}\left(k, q_{x}\right)}{\sqrt{k^{2}+q_{x}^{2}}}
$$

where $\varepsilon$ is the static dielectric constant of the semiconductor and $F_{i j l m}\left(k, q_{x}\right)$ is the form factor, which takes into account the finite thickness of the quantum wire and is given by

$$
F_{i j l m}\left(k, q_{x}\right)=\int d y \int d y^{\prime} \phi_{i}^{*}(y) \phi_{j}(y) \exp \left(-\sqrt{k^{2}+q_{x}^{2}}\left|y-y^{\prime}\right|\right) \phi_{l}^{*}\left(y^{\prime}\right) \phi_{m}\left(y^{\prime}\right)
$$

where $\phi_{j}(y)$ is the wave function of the electrons confined by the parabolic potential. We have calculated the ground state intrasubband (0-0) pair-correlation function within the Random-Phase Approximation for $\mathrm{GaAs}-\mathrm{Al}_{x} \mathrm{Ga}_{1-x} \mathrm{As}$ parabolic quantum well wires as a function of the electronic density for two energy separation of the subbands, $\hbar \Omega=2.0$ $\mathrm{meV}$ and $\hbar \Omega=2.5 \mathrm{meV}$.

Fig. 1 shows the intrasubband (0-0) pair-correlation function, $g(x)$, as a function of the dimensionless interparticle separation coordinate $x$, which is written in units of the effective Bohr radius, $a_{0}^{*}=101$, for three electronic density values $\rho=2.45 \times 10^{5} \mathrm{~cm}^{-1}, \rho=3.19 \times 10^{5}$ $\mathrm{cm}^{-1}$ and $\rho=4.91 \times 10^{5} \mathrm{~cm}^{-1}$ for a subband energy separation $\hbar \Omega=2.5 \mathrm{meV}$. It can be seen that the RPA intrasubband pair-correlation function always assumes positive values for any interparticle separation for the electronic densities $\rho=3.19 \times 10^{5} \mathrm{~cm}^{-1}$ and $\rho=4.91 \times 10^{5} \mathrm{~cm}^{-1}$. The critical density in this case is $\rho=3.19 \times 10^{5} \mathrm{~cm}^{-1}$, since decreasing the separation between two particles the pair correlation function decreases going to the value zero in the limit of $x \rightarrow 0$, but never assuming negative values. For smaller values than the critical density the RPA paircorrelation function assumes negative results for small values of the interparticle separation, as one can observe from Fig. 1 for the electronic density $\rho=2.45 x 10^{5} \mathrm{~cm}^{-1}$.

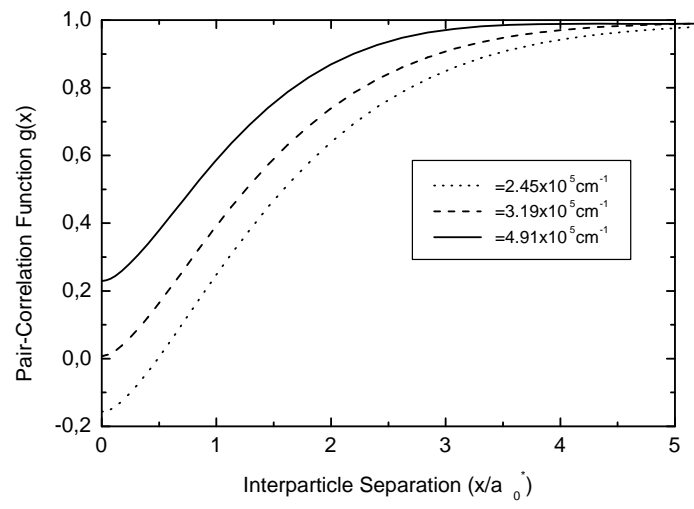

Figure 1. The intrasubband (0-0) pair-correlation function, $g(x)$, as a function of the dimensionless interparticle separation coordinate $x$, for three electronic density values, $\rho=2.45 \times 10^{5} \mathrm{~cm}^{-1}$, $\rho=3.19 x 10^{5} \mathrm{~cm}^{-1}$ and $\rho=4.91 \times 10^{5} \mathrm{~cm}^{-1}$.

The intrasubband (0-0) pair-correlation function for particles near the origin of the coordinates system $(x \approx 0)$ as a function of the electronic density for wires with subband separation energies $\hbar \Omega=2.0 \mathrm{meV}$ and $\hbar \Omega=2.5 \mathrm{meV}$ are shown in Fig. 2. As one can see from that figure, the critical density value decreases with decreasing subband energy separation, and for the wire with $\hbar \Omega=2.0 \mathrm{meV}$ the critical density is $\rho=3 x 10^{5} \mathrm{~cm}^{-1}$. The fact of the RPA paircorrelation function have only positive values for electronic densities higher than the critical density does not guarantee that the local field correction can be neglected. The negative signal of the pair-correlation function for small interparticle separation gives us just the electronic density regions where the RPA approach cannot be used.

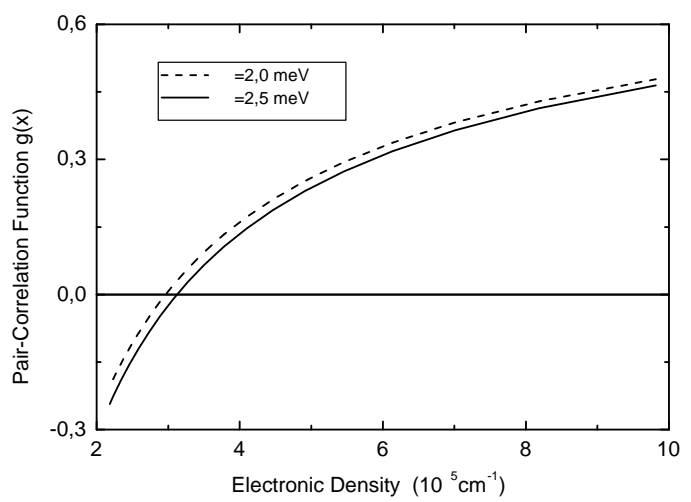

Figure 2. The intrasubband (0-0) pair-correlation function for particles near the origin of the coordinates system $(x \approx 0)$ as a function of the electronic density for wires with subband separation energies $\hbar \Omega=2.0 \mathrm{meV}$ and $\hbar \Omega=2.5 \mathrm{meV}$.

In conclusion, we have calculated the intrasubband (0-0) pair-correlation function for a quasi-one-dimensional electron gas confined in a $\mathrm{GaAs}-\mathrm{Al}_{x} \mathrm{Ga}_{1-x}$ As parabolic QWW within the Random-Phase Approximation. We have studied the dependence of the pair-correlation function on the electronic density, and found the regions where the RPA approach gives physically acceptable results, i. e., we found the electronic density values where $g(x)$ assume positive values for small interparticle separation. We considered 
two wires with subband energy separation $\hbar \Omega=2.0 \mathrm{meV}$ and $\hbar \Omega=2.5 \mathrm{meV}$ and the values $\rho=3 x 10^{5} \mathrm{~cm}^{-1}$ and $\rho=3.19 \times 10^{5} \mathrm{~cm}^{-1}$ for the critical densities were found. Therefore, the local field correction (LFC) must always be used for density values lower than the critical density, and for higher electronic densities it gives an important contribution that cannot be neglected.

\section{Acknowledgement}

We thank to the Fundação de Apoio à Pesquisa FUNAPE - UFG for financial support.

\section{References}

[1] T. Demmel, D. Heitmann, P. Grambow, and K. Ploog, Phys. Lett. 66, 2657 (1991).

[2] A. N. Borges, S. A. Leão, and O. Hipólito, Phys. Rev. B 55, 4680 (1997).

[3] A. N. Borges, M. H. Degani, and O. Hipólito, Superlattices and Microstructures, 13, 1, 51 (1993).

[4] V. B. Campos, M. H. Degani, and O. Hipólito, Superlattices and Microstructures, 17, 1, 85 (1995).

[5] L. Camels and A. Gold, Solid State Commun. 92, 619 (1994).

[6] P. C. M. Machado, F. A. P. Osório, and A. N. Borges, Modern. Phys. Lett. B11, 441 (1997). 